\title{
An application of Taguchi's method to robust design of aircraft structures
}

\author{
S. Hernández \& J. Díaz \\ School of Civil Engineering, University of Coruña, La Coruña, Spain
}

\begin{abstract}
The purpose of this work is to demonstrate the application of a robust design procedure to the design of aircraft structures. Taguchi's method of robust design has been applied to obtain the most appropriate values of a set of control factors considering several noise factors. The goal is to maximize the robustness of the design and decrease the loss of quality. A description of the methodology is provided and an application example is solved, using a selected set of noise and control factors, to describe the suitability of this technique for structural design in problems where inaccuracies are present. The results show an improvement in the response of the components, maintaining a low dependence on input value variations and producing designs that increase the quality level and enhance the robustness. Other different sets can be defined, as the method is completely general.

Keywords: robust design, Taguchi methods, aircraft structures.
\end{abstract}

\section{Introduction}

Robust analysis improves the capabilities of uncertainty quantification techniques because considers a function representing robustness, which is optimized in the procedure.

Structural problems in aerospace engineering take place in an environment where many parameters required for the mathematical formulation of the problem do not have precise numerical values and some degree of uncertainty or inaccuracy exists. A deterministic analysis may be unable to provide the amount of information required by designers and thus other approaches taking into account uncertainties are necessary. That circumstance also requires for designers to be aware about the variations on the performance of prototypes, 
considering these random variables. Besides, an aim of robustness or, in other words, the idea of choosing designs that reduce the uncertainty of the performance is preferred $[1,2]$.

Robustness is the aim of obtaining a design that is, as less as possible, insensitive to variation in the input parameters [3]. In robust analysis, given a target value for a design response, the preferred candidate is not the one closest to the target value, but the one least sensitive to parameter variations.

Robust analysis improves the capacity of uncertainty quantification techniques because it incorporates a function representing robustness, which is optimized in the procedure. The flowchart of this methodology is shown in Figure 1, where a product or process governed by a certain number of control factors and subjected to uncertainty coming from noise factors, is designed to achieve a response with the least possible dispersion.

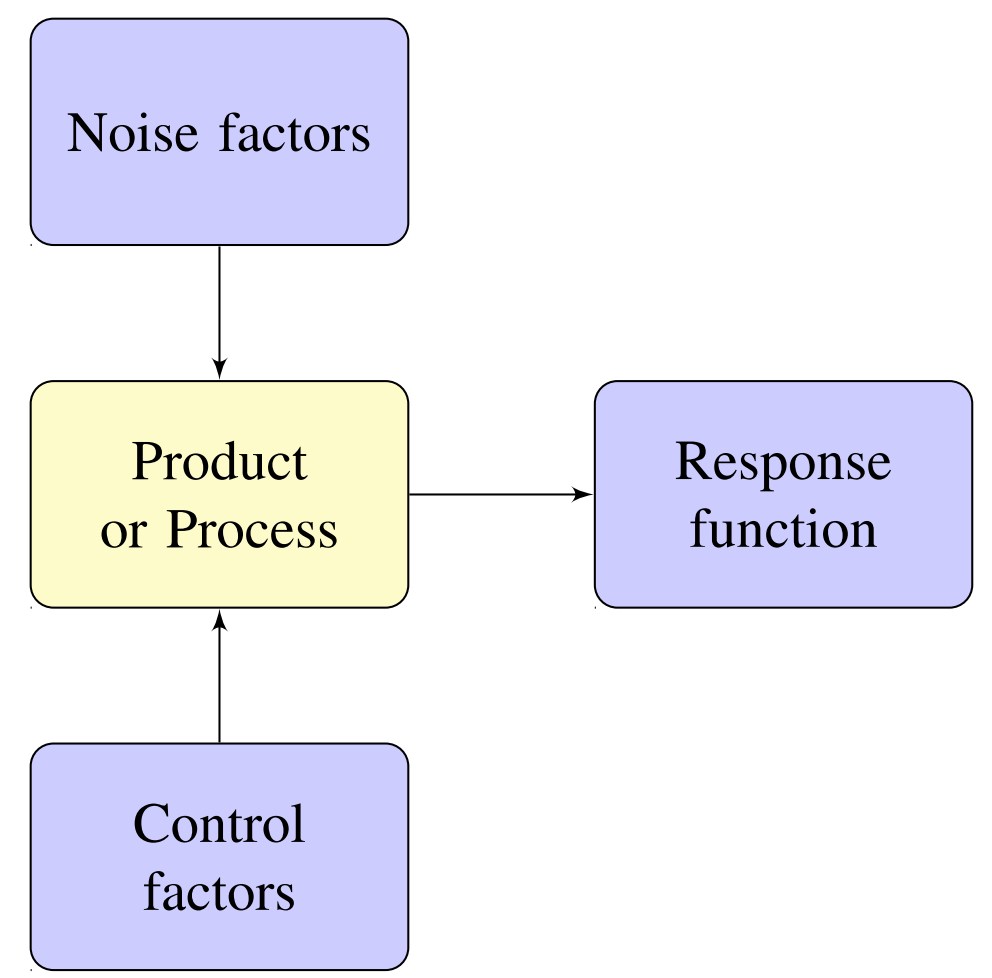

Figure 1: Flowchart of the robust design procedure.

\section{Robust design by the Taguchi's method}

In Taguchi's method, robustness of a design is determined by the value of a function defining the quality loss of the design and the best design is the one having the lowest quality loss. Taguchi's method formulates the robust design 
problem in an uncertain environment considering three concepts: two sets of factors and a preferred response. The first set is formed by the noise factors, which are parameters related to the problem with a range of values not controlled by the designer and constituting the source of uncertainty. The second set includes the control factors, which are parameters related to the problem having a range of values that can be selected by the designer. The third concept is a response of the system, with a preferred value chosen by the designer, which is the target of the problem. Any difference between such value and the performance of a given design is considered to be a decrease in its adequacy. In that regard, the so-called quality loss function is defined to measure such circumstances. The strategy of Taguchi's method for finding a robust design is to identify the proper values of the control factors that minimize the quality loss function, while taking into account the variability of the noise factors. To summarize, the steps of the method are the following [4]:

1. Define the vector of noise factors and their levels.

2. Define the vector of control factors and their levels.

3. Define the orthogonal arrays, which are related to the number of experiments to perform

4. Carry out the analyses defined by the orthogonal arrays.

5. Define the quality loss function.

6. Observe the effect of each control factor in the quality loss function by using analysis of mean method (ANOM).

7. Identify the best level of each control factor, and therefore the most robust design.

Three different classes of problems can be solved by Taguchi's method [4]:

a) Problems aiming a fixed value for the response, which are called nominal the best type problems (NTB).

b) Problems aiming for the lowest possible value, called smaller the better type problems (STB).

c) Problems aiming for the greatest possible value of the response. They are called larger the better type problems (LTB).

\section{Application example: stiffened panel for aircraft fuselage}

Taguchi methods have been applied in different field of aircraft engineering, amongst them in thermal problems, in which uncertainties related to several characteristics of the problem formulation need to be considered [5]. In this paper, an application on structural mechanics has been selected. The work performed consists in the application of a robust design procedure to the design of an aircraft structure. Taguchi's method of robust design has been applied to obtain the most appropriate values of a set of control factors considering several noise factors. The goal is to maximize the robustness of the design and decrease the loss of quality.

The application example is a curved stiffened composite panel (Figure 1), where the specific uncertainties related to this typology, such as curvature, composite lay-up and mechanical properties are taken into account. 
A finite element mesh is built with four node shell elements to model the skin, and with beam elements for the frames and the stiffeners, resulting in 8526 degrees of freedom. The loads applied consist of shear and in plane compression components. Buckling factor $\lambda$ (Figure 2) is selected to define the design quality level (Figure 3).

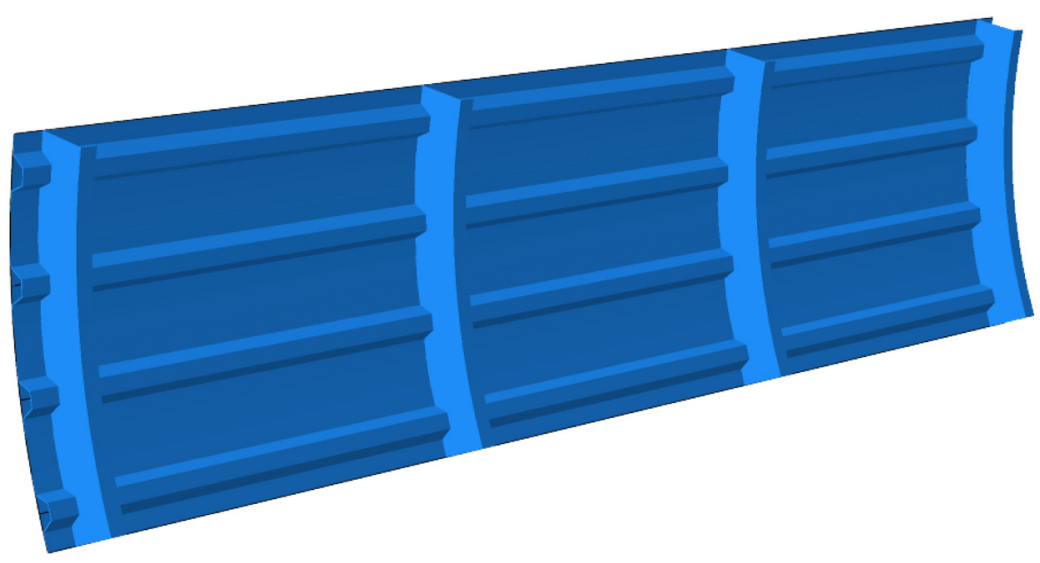

Figure 2: Geometry of the panel.

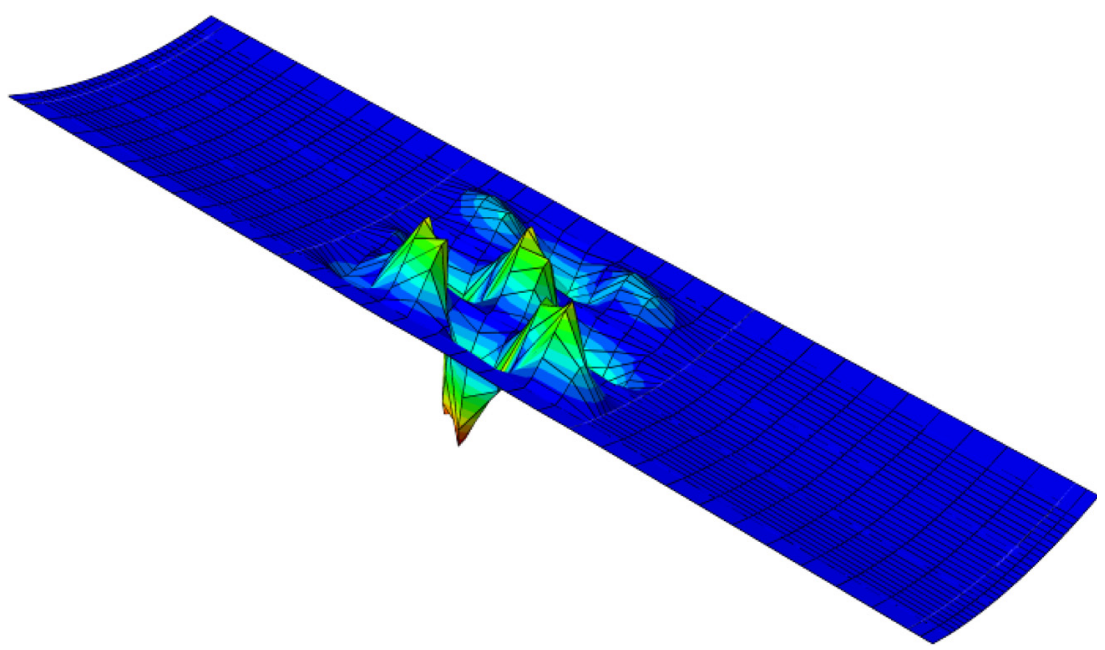

Figure 3: Buckling mode.

Seven noise factors are included in the study: aluminium modulus of elasticity, composite $\mathrm{E}_{1}$ and $\mathrm{E}_{2}$ elastic moduli, the percentage of 0 and 90 degree plies of the skin and the values of compression and shear load. These factors 
have a mean value $\mu$ and a percentage of variation $v$. From these two statistical parameters, the value of standard deviation $\sigma$ can be evaluated as:

$$
\sigma=\frac{\mu \nu}{3}
$$

For each noise factor, two different values, namely $\mu-\sigma$ and $\mu+\sigma$, are considered in the study. According to terminology used by Taguchi, these two values will be referred to as level 1 and level 2 . The information for the complete definition of noise factors appears in Table 1.

Table 1: $\quad$ Noise factors and their levels.

\begin{tabular}{lcccccccc}
\hline Noise factor & Number & Symbol & Units & $\mu$ & $\nu$ & $\sigma$ & Level 1 & Level 2 \\
\hline Aluminium modulus of elasticity & 1 & $E_{a l u}$ & $G P a$ & 72 & 0.3 & 7.2 & 64.8 & 79.2 \\
Composite $E_{1}$ & 2 & $E_{1}$ & $G P a$ & 157 & 0.3 & 15.7 & 141.3 & 172.7 \\
Composite $E_{2}$ & 3 & $E_{2}$ & $G P a$ & 8.5 & 0.3 & 0.85 & 7.65 & 9.35 \\
Skin percentage 0-degree plies & 4 & $S P_{0}$ & $\%$ & 25 & 0.3 & 2.5 & 22.5 & 27.5 \\
Skin percentage 90-degree plies & 5 & $S P_{90}$ & $\%$ & 25 & 0.3 & 2.5 & 22.5 & 27.5 \\
Compression load & 6 & $L_{C}$ & $M P a$ & 1.0 & 0.3 & 0.1 & 0.9 & 1.1 \\
Shear load & 7 & $L_{S}$ & $M P a$ & 0.5 & 0.3 & 0.05 & 0.45 & 0.55 \\
\hline
\end{tabular}

In the case of control factors, four different characteristics of the component are chosen: skin thickness, frame height, stiffener height and panel radius. In each of them, up to three different levels are selected, which are shown in Table 2.

Table 2: $\quad$ Control factors and their levels.

\begin{tabular}{lcccccc}
\hline Control factor & Number & Symbol & Units & Level 1 & Level 2 & Level 3 \\
\hline Skin thickness & 1 & $S_{k t}$ & $m m$ & 1.8 & 2.0 & 2.2 \\
Frame height & 2 & $F_{H}$ & $m m$ & 74.7 & 83.0 & 91.3 \\
Stiffener height & 3 & $S_{H}$ & $m m$ & 22.5 & 25.0 & 27.5 \\
Panel radius & 4 & $P_{R}$ & $m m$ & 1810.8 & 2012 & 2213.2 \\
\hline
\end{tabular}

After the noise and control factors are selected, the next step is to define the set of orthogonal arrays. The idea is to predict a set of analysis unbiasing the significance of any noise factors and, therefore, to assign the same number of events to all of them. Table 3 shows an orthogonal array L8 $\left(2^{7}\right)$ containing the levels selected for each noise factor. Each one of the eight combinations is called experiment in Taguchi's terminology. In the same way, an orthogonal array L9 $\left(3^{4}\right)$ needs to be defined for control factors and so Table 4 describes the levels selected for the nine experiments. 
Table 3: $\quad$ Orthogonal array of noise factors.

\begin{tabular}{cccccccc}
\hline & \multicolumn{8}{c}{ Noise factor } \\
Experiment & \multicolumn{1}{c}{${ }^{*}$} & 2 & 3 & 4 & 5 & 6 & 7 \\
\hline 1 & 1 & 1 & 1 & 1 & 1 & 1 & 1 \\
2 & 1 & 1 & 1 & 2 & 2 & 2 & 2 \\
3 & 1 & 2 & 2 & 1 & 1 & 2 & 2 \\
4 & 1 & 2 & 2 & 2 & 2 & 1 & 1 \\
5 & 2 & 1 & 2 & 1 & 2 & 1 & 2 \\
6 & 2 & 1 & 2 & 2 & 1 & 2 & 1 \\
7 & 2 & 2 & 1 & 1 & 2 & 2 & 1 \\
8 & 2 & 2 & 1 & 2 & 1 & 1 & 2 \\
\hline
\end{tabular}

Table 4: $\quad$ Orthogonal array of control factors.

\begin{tabular}{ccccc}
\hline \multirow{2}{*}{ Experiment } & \multicolumn{4}{c}{ Control factor } \\
& 1 & 2 & 3 & 4 \\
\hline 1 & 1 & 1 & 1 & 1 \\
2 & 1 & 2 & 2 & 2 \\
3 & 1 & 3 & 3 & 3 \\
4 & 2 & 1 & 2 & 3 \\
5 & 2 & 2 & 3 & 1 \\
6 & 2 & 3 & 1 & 2 \\
7 & 3 & 1 & 3 & 2 \\
8 & 3 & 2 & 1 & 3 \\
9 & 3 & 3 & 2 & 1 \\
\hline
\end{tabular}

It should be kept in mind that, for each experiment included in the array of control factors, the eight cases considered in the orthogonal array of noise factors need to be carried out, which results in seventy two solutions. Each of them corresponds to different cases of the structural problem, therefore up to seventy two structural analyses with the numerical values of the levels of noise and control factors used in the study were performed. For all of them, the buckling factor is obtained.

Using those results, a nominal the best type problem is formulated. In this variant of Taguchi's method, buckling factor is selected as target of the design. The problem will be formulated aiming to minimize variations of $\lambda$ due to noise 
factors. As the variation is an indication of quality loss, the problem represents the lower the difference, the better the behaviour. Therefore, this formulation corresponds to the definition of nominal the best type. According to this approach, a signal to noise $(\mathrm{S} / \mathrm{N})$ function $\eta$ is defined as follows:

$$
\eta=10 \log _{10}\left(\frac{\mu_{t}^{2}}{\sigma_{t}^{2}}\right)
$$

In the expression above, $\mu_{\mathrm{t}}$ is the mean value and $\sigma_{\mathrm{t}}$ is the standard deviation of the buckling factor in the experiments associated to the orthogonal array of control factors. It can be observed that function $\eta$ includes the standard deviation in the denominator, which means that the lower the value of $\sigma_{t}$ the greater the value of $\eta$, and so the quality of the design. Therefore, minimization of quality losses can be interpreted as maximization of function $\eta$.

After the calculation of each set of eight structural analyses for each control factor experiment, the values of $\eta$ are shown in Table 5. Then, the analysis of mean must be carried out. In this step, the mean value of $\eta$ for the three experiments corresponding to the same level of each control factor is evaluated. Table 6 shows the complete set of values. Numerical results of ANOM can be shown graphically and the values corresponding to buckling factor appear in Figure 4. It is already known that maximizing $\eta$ is equivalent to decreasing quality losses. Therefore Figure 4 clearly indicates which level of each control factor produces the best values of $\eta$ and thus the set of best levels can be easily chosen.

Table 5: $\quad$ Signal to noise ratio.

\begin{tabular}{|c|c|c|c|c|c|}
\hline \multirow{2}{*}{ Experiment } & \multicolumn{4}{|c|}{ Control factor } & \multirow[t]{2}{*}{$\eta$} \\
\hline & 1 & 2 & 3 & 4 & \\
\hline 1 & 1 & 1 & 1 & 1 & -6.162 \\
\hline 2 & 1 & 2 & 2 & 2 & -6.109 \\
\hline 3 & 1 & 3 & 3 & 3 & -6.089 \\
\hline 4 & 2 & 1 & 2 & 3 & -8.203 \\
\hline 5 & 2 & 2 & 3 & 1 & -8.612 \\
\hline 6 & 2 & 3 & 1 & 2 & -8.132 \\
\hline 7 & 3 & 1 & 3 & 2 & -10.470 \\
\hline 8 & 3 & 2 & 1 & 3 & -9.658 \\
\hline \multirow[t]{2}{*}{9} & 3 & 3 & 2 & 1 & -10.414 \\
\hline & & & & & -8.205 \\
\hline
\end{tabular}


10 High Performance Structure and Materials VI

Table 6: Analysis of mean results for nominal the best type problem.

\begin{tabular}{cccc}
\hline \multirow{2}{*}{ Control factor } & \multicolumn{4}{c}{ Level } \\
& 1 & 2 & 3 \\
\hline 1 & -6.120 & -8.316 & -10.181 \\
2 & -8.278 & -8.126 & -8.211 \\
3 & -7.984 & -8.242 & -8.390 \\
4 & -8.396 & -8.237 & -7.983 \\
\hline
\end{tabular}

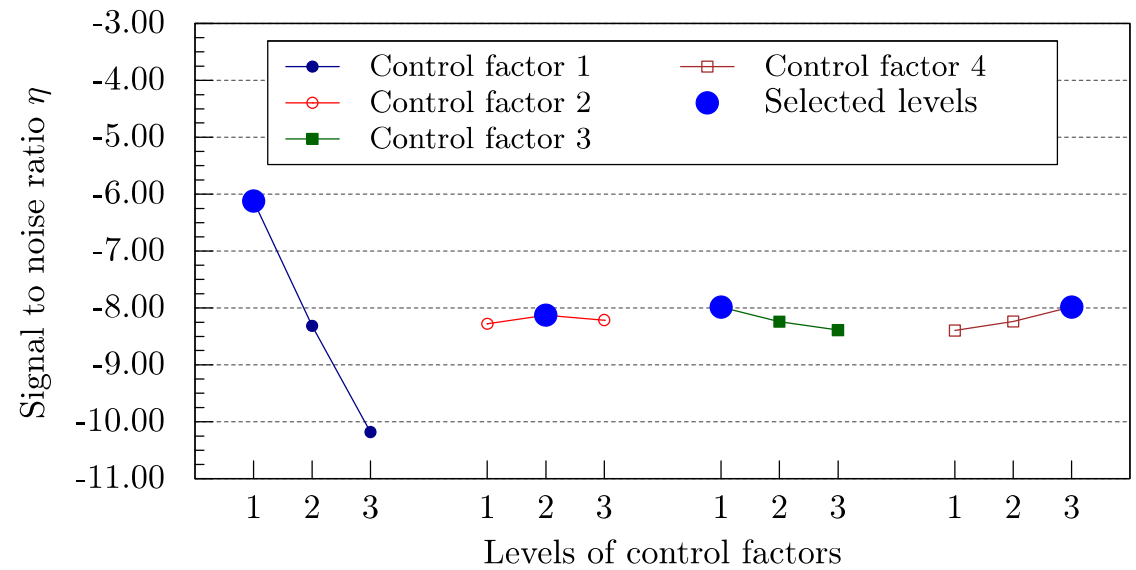

Figure 4: $\quad$ Results of ANOM and selected levels of control factors.

It should be mentioned that the combination of control factor levels corresponding to robust design does not exist in the orthogonal array of Table 4. This feature is one of the powerful characteristics of Taguchi's method, as it can identify combinations of control factors with better performance than those included in the set of experiments evaluated. The last step in robust design is to confirm that the set of levels selected behaves better than the set of experiments carried out. This phase is called experiment verification and is accomplished by comparing the mean value and the standard deviation of the seventy two experiments defined by the orthogonal arrays of control and noise factors, named starting condition, and the mean value and the standard deviation corresponding to the eight experiments carried out considering the selected set of control factors at robust design and the orthogonal array of noise factors. Table 7 shows the numerical values obtained. It can be concluded that $\eta$ has a larger value, meaning lower quality losses, with an improvement of $40.9 \%$. 
Table 7: $\quad$ Verification results.

\begin{tabular}{cccc}
\hline $\begin{array}{l}\text { Initial } \\
\text { design }\end{array}$ & $\begin{array}{c}\text { Robust } \\
\text { design }\end{array}$ & $\begin{array}{c}\text { Improvement } \\
(\%)\end{array}$ \\
\hline$\eta$ & -8.205 & -5.821 & 40.938 \\
\hline
\end{tabular}

\section{Conclusions}

Several conclusions can be extracted from this research:

1) Taguchi's method is suitable to obtain robust designs in structural problems. The method proceeds by carrying out a discrete search in the range of variation of noise factors and control factors and so the cost of application, mainly the computational resources required to solve the problems is reduced.

2) Three formulations can be defined: nominal the best, smaller the better and larger the better. The procedure to be used depends of the objective of the problem.

3) An application example corresponding to the design of a composite stiffened panel for aircraft fuselage has been solved using the nominal the best procedure to demonstrate the methodology using a selected set of noise and control factors, obtaining an improved design with an increased level of robustness.

4) As the method is totally general, other different sets could be defined and more complex formulations, including more structural considerations could be implemented.

\section{Acknowledgement}

This research has been funded by the Spanish Ministry of Science and Innovation under project DPI2010-16238.

\section{References}

[1] G. Park, T.-H. Lee, K.H. Lee, K.-H Hwang. Robust design: An overview. AIAA Journal 44 (1) (2006) $181-191$.

[2] G. Taguchi, S. Chowdhury, Y. Wu, S. Taguchi, H. Yano. Taguchi's quality engineering handbook. John Wiley and Sons - ASI Consulting Group, Hoboken, NJ, 2005.

[3] G. Taguchi, S. Chowdhury, S. Taguchi. Robust engineering. McGraw-Hill, New York, 2000. 
12 High Performance Structure and Materials VI

[4] M. S. Phadke, Quality engineering using robust design, Prentice Hall, Englewood Cliffs, NJ, 1989.

[5] J. Díaz, S. Hernández, Uncertainty quantification and robust design of aircraft components under thermal loads, Aerospace Science and Technology, Vol. 14, Number 8, pp 527-534, 2010. 